\title{
Scalar gravitation: A laboratory for numerical relativity
}

\author{
Stuart L. Shapiro and Saul A. Teukolsky \\ Center for Radiophysics and Space Research \\ and Departments of Astronomy and Physics, Cornell University, Ithaca, New York 14853
}

(Received 9 October 1992)

\begin{abstract}
While not a correct physical theory, relativistic scalar gravitation provides a simple test site for developing many of the tools of numerical relativity. In contrast with general relativity, scalar gravitation allows gravitational waves to be generated in spherical symmetry. Hence one needs only one spatial dimension to try out methods of calculating wave emission and propagation. Using this theory, we have built a mean-field particle simulation scheme to study the dynamical behavior of collisionless matter in spherical symmetry. We find that we are able to calculate smooth and accurate wave forms, despite the stochastic representation of the matter source terms caused by sampling with a finite number of particles. A similar scheme should provide accurate wave forms in general relativity, provided sufficient computer resources are used.
\end{abstract}

PACS number(s): 04.30. $+\mathrm{x}, 02.70 . \mathrm{Bf}, 03.50 . \mathrm{Kk}$

\section{INTRODUCTION}

Numerical relativity is the most promising approach for finding solutions of Einstein's equations that describe realistic physical phenomena. While a number of important results have been obtained by numerical means, the field is beset by many technical difficulties. In addition to the usual problems of solving multidimensional partial differential equations numerically, relativity presents some unique complications. One of these is dealing with the appearance of singularities and black holes, which frequently form during gravitational collapse. The development of a singularity, for example, forces a numerical simulation to terminate. Even if all singularities are hidden inside black holes, it may be impossible to follow the evolution in the exterior regions once a singularity is present. The general approach to dealing with this problem is to exploit the gauge freedom of the theory to choose coordinates that hold back the formation of singularities in strong field regions, allowing the exterior evolution to proceed to arbitrarily late times. However, there is no recipe for finding a suitable set of coordinates to accomplish this in general. Another complication in numerical relativity is extracting information about gravitational waves when the wave amplitudes are typically much smaller than the background gravitational field variables.

Developing and testing new algorithms for tackling numerical relativity is difficult because the human and computational resources required to simulate even the simplest dynamical problems can be considerable. For example, to generate gravitational waves one must deal with nonspherical systems, which requires at least two spatial dimensions plus time. As a result, the system of equations is sufficiently complicated that experimenting with different coordinate choices or algebraic formulations requires an enormous investment. Furthermore, the required computer resources often tax the largest supercomputers.
One of the purposes of this paper is to study a simpler theory of relativistic gravitation to learn how to deal with some of these issues. The theory we consider describes gravitation by a nonlinear scalar gravitational field in special relativity and is presented in exercise 7.1 of Ref. [1]. Of course, this theory disagrees violently with experiment, giving incorrect predictions for the three classical tests of general relativity. Our purpose, however, is to use the theory to develop numerical tools that can then be applied to solve problems in general relativity. In addition, by studying scalar gravitation we can examine some of the generic features of a nonlinear dynamical theory of gravitation in a simple setting, such as gravitational collapse and wave production.

One of the obvious advantages of scalar gravitation is that fewer variables are needed to describe the field. The theory also admits a local law of energy conservation, while general relativity only obeys global energy conservation. In numerical work, such a conservation law provides a strong check on the accuracy of an integration. More significantly, scalar gravitational waves can be generated even in spherical symmetry. This means that a numerical code need only deal with one spatial dimension plus time to study wave generation and propagation, a considerable simplification over general relativity.

Scalar gravitation does not address all of the issues that arise in numerical relativity. For example, because the metric is always Minkowski type, coordinate singularities related to the gauge freedom of general relativity do not arise. To follow a dynamical evolution in scalar gravitation, one need only solve a single hyperbolic equation, while in general relativity there are coupled elliptic equations as well as hyperbolic equations. No black holes occur in scalar gravitation to complicate the dynamics.

A specific computational issue that we address in this paper is prompted by our recent mean-field particle simulations of collisionless matter in general relativity [2]. In these simulations, which obeyed global energy conservation to high accuracy, the emitted gravitational radiation 
carried off only a small fraction of the total energy. However, the computed wave forms turned out to be much noisier than expected. The obvious concern is whether it is inherent in a particle simulation scheme, with its stochastic representation of the smooth particle distribution, that the computed waveforms are always noisy. Alternatively, it is conceivable that while our simulations used enough particles to track the global dynamics accurately, they sampled too few to get smooth waveforms. There may also exist better particle binning algorithms for the matter source terms $\left(T_{\mu \nu}\right)$, which will yield smoother waveforms with smaller numbers of particles. By constructing a mean-field particle simulation scheme to evolve collisionless matter in scalar gravitation theory, we can address this question while working only in spherical symmetry.

The principal conclusion of this paper is that there is no intrinsic reason preventing a mean-field particle simulation from calculating smooth and accurate gravitational waveforms. By using enough particles per spatial grid zone, one can compute reasonably accurate wave forms. Adopting a better binning algorithm gives even smoother and more reliable results.

Scalar gravitation is a good laboratory not just for testing codes that handle collisionless matter, but also for codes that treat hydrodynamics. We give the relevant equations in an Appendix.

\section{BASIC EQUATIONS}

The scalar gravitational field theory that we wish to explore is presented in exercise 7.1 of Ref. [1]. The gravitational field is described by a massless scalar field $\Phi\left(x^{\alpha}\right)$ in special relativity. The scalar field does not modify the background spacetime geometry, which is always Minkowskian. Consider a particle of mass $m$ moving along a world line $z^{\alpha}(\lambda)$. Let $\dot{z}^{\alpha}$ denote $d z^{\alpha} / d \lambda$. Then the action for the field-particle system is

$$
I=\int \mathcal{L}(-g)^{1 / 2} d^{4} x,
$$

where the Lagrangian density $\mathcal{L}$ is

$$
\mathcal{L}=-\frac{1}{8 \pi G} g^{\alpha \beta} \Phi_{, \alpha} \Phi_{, \beta}-\rho e^{\Phi},
$$

and where the comoving density is

$\rho=m \int\left(-g_{\alpha \beta} \dot{z}^{\alpha} \dot{z}^{\beta}\right)^{1 / 2} \delta^{4}[\vec{x}-\vec{z}(x)](-g)^{-1 / 2} d \lambda$.

Here the metric tensor $g_{\alpha \beta}$ is the usual Minkowski metric $\eta_{\alpha \beta}$, since spacetime is flat in this theory [i.e., $\eta_{\alpha \beta}=\operatorname{diag}(-1,1,1,1)$ in Cartesian coordinates]. We use arrows to denote four-vectors and boldface to denote three-vectors. We will generally set the speed of light $c=1$, but will display the gravitational coupling constant (Newton's constant) $G$ explicitly. If we choose $\lambda$ equal to the proper time $\tau$ along the particle world line, then

$$
\begin{aligned}
\rho & =m \int \delta^{4}[\vec{x}-\vec{z}(\tau)](-g)^{-1 / 2} d \tau \\
& =m \delta^{3}[\mathbf{x}-\mathbf{z}(t)](-g)^{-1 / 2} / \gamma,
\end{aligned}
$$

where $\gamma=\dot{z}^{0}$ is the usual Lorentz factor.
Varying the Lagrangian (2.2) with respect to $\Phi$ gives the field equations of motion

$$
\square \Phi=4 \pi G e^{\Phi} \rho \text {. }
$$

In the Newtonian limit, where $\Phi \ll 1$, Eq. (2.5) becomes linear and reduces to Poisson's equation. Varying the Lagrangian with respect to $z^{\alpha}$ gives the particle equations of motion:

$$
\frac{D^{2} z^{\alpha}}{d \tau^{2}}+\left[g^{\alpha \beta}+\frac{d z^{\alpha}}{d \tau} \frac{d z^{\beta}}{d \tau}\right] \Phi_{, \beta}=0,
$$

where $D$ denotes covariant differentiation. Here we are allowing for curvilinear coordinates; covariant differentiation reduces to ordinary differentiation in Cartesian coordinates. In the nonrelativistic limit, Eq. (2.6) implies that the gravitational force is $-\nabla \Phi$. The fully relativistic form ensures that the four-velocity $u^{\alpha}=d z^{\alpha} / d \tau$ remains orthogonal to the four-acceleration $a^{\alpha}=D u^{\alpha} / d \tau$.

Matter conservation is expressed by the condition

$$
\nabla \cdot \vec{J}=0
$$

where the components of the matter current density are

$$
J^{\alpha}=m \int u^{\alpha} \delta^{4}[\vec{x}-\vec{z}(\tau)](-g)^{-1 / 2} d \tau,
$$

i.e.,

$$
J^{0}=\gamma \rho, \quad J^{i}=\gamma \rho v^{i},
$$

where $v^{i}$ is the usual three-velocity. Integrating Eq. (2.7) over all space yields a conserved rest mass:

$$
M_{0}=\int \gamma \rho d^{3} x=\text { const . }
$$

Conservation of energy-momentum follows from

$$
\nabla \cdot \mathrm{T}=0,
$$

where $\mathbf{T}$ is the total stress-energy tensor of the system:

$$
T^{\mu \nu}=\frac{2}{(-g)^{1 / 2}} \frac{\delta\left[\mathcal{L}(-g)^{1 / 2}\right]}{\delta g_{\mu \nu}} .
$$

Carrying out the variation using Eq. (2.2), we find

$$
T_{\mu \nu}=T_{\mu \nu}^{\mathrm{field}}+T_{\mu \nu}^{\mathrm{particle}},
$$

where

$$
\begin{aligned}
& T_{\mu \nu}^{\mathrm{field}}=\frac{1}{4 \pi G}\left[\Phi_{, \mu} \Phi_{, \nu}-\frac{1}{2} g_{\mu \nu} \Phi^{, \alpha} \Phi_{, \alpha}\right], \\
& T_{\mu \nu}^{\text {particle }}=\rho e^{\Phi} u_{\mu} u_{\nu} .
\end{aligned}
$$

One can check that Eq. (2.14) satisfies Eq. (2.11), using Eqs. (2.5) and (2.6). Note that Eq. (2.5) can be written

$$
\square \Phi=-4 \pi G T,
$$

where $T=g^{\mu \nu} T_{\mu \nu}^{\text {particle }}$ is the trace of the particle stressenergy tensor. Equation (2.15) applies also for other matter sources, where $T$ is the trace of the appropriate matter stress-energy tensor.

The above equations are easily extended to describe a 
collection of particles. Simply let

$$
m \rightarrow \sum_{A} m_{A}, \quad u^{\mu} \rightarrow u_{A}^{\mu}, \text { etc. }
$$

Treated in this way, the matter consists of a swarm of collisionless particles. In the limit of a large number of particles of infinitesimal rest mass, the matter can be characterized by a smooth phase space distribution function satisfying the Vlasov equation. Tracking the world lines of these particles by integrating Eq. (2.6) in the mean background potential is equivalent to solving the Vlasov equation for the matter in a scalar gravitational field.

Alternatively, the above equations can be adapted to describe fluid matter. The equations of hydrodynamics for an ideal fluid in this theory are given in the Appendix.

A general dynamical system is characterized by a time-varying matter density and velocity profile, interacting with a time-varying scalar field containing radiation. Equation (2.11) gives rise to a particularly simple conserved integral for the total energy at any time $t$ inside a sphere of radius $r$ centered at the origin:

$$
\begin{gathered}
\int_{0}^{r} r^{2} d r \int d \Omega\left[\frac{1}{8 \pi G}\left(\Phi_{, 0}^{2}+(\nabla \Phi)^{2}\right)+\rho e^{\Phi} \gamma^{2}\right] \\
-r^{2} \int_{0}^{t} d t \int d \Omega\left[\frac{1}{4 \pi G} \Phi_{, 0} \Phi_{, r}-\rho e^{\Phi} \gamma^{2} v_{r}\right] \\
\quad=\int_{0}^{r} r^{2} d r \int d \Omega\left[\frac{1}{8 \pi G}\left(\Phi_{, 0}^{2}+(\nabla \Phi)^{2}\right)+\rho e^{\phi} \gamma^{2}\right]_{t=0} .
\end{gathered}
$$

The first term in Eq. (2.17) is the volume integral of the total energy density $T^{00}$. The second term is the outgoing flux $T^{0 r}$ of matter and field energy through the surface of the sphere, integrated over time. The right-hand side is the volume integral of the initial total energy density. For computational purposes, it is convenient to combine Eq. (2.17) with the continuity equation (2.7), which can be integrated over the sphere to give

$$
\begin{aligned}
\int_{0}^{r} r^{2} d r \int d \Omega \gamma \rho+r^{2} \int_{0}^{t} d t & \int d \Omega \gamma \rho v_{r} \\
& =\int_{0}^{r} r^{2} d r \int d \Omega[\gamma \rho]_{t=0} .
\end{aligned}
$$

Subtracting Eq. (2.18) from Eq. (2.17) gives

$$
\begin{array}{r}
\int_{0}^{r} r^{2} d r \int d \Omega\left[\frac{1}{8 \pi G}\left(\Phi_{, 0}^{2}+(\nabla \Phi)^{2}\right)+\rho \gamma\left(e^{\Phi} \gamma-1\right)\right]-r^{2} \int_{0}^{t} d t \int d \Omega\left[\frac{1}{4 \pi G} \Phi_{, 0} \Phi_{, r}-\gamma \rho v_{r}\left(e^{\Phi} \gamma-1\right)\right]_{t=0} \\
=\int_{0}^{r} r^{2} d r \int d \Omega\left[\frac{1}{8 \pi G}\left(\Phi_{, 0}^{2}+(\nabla \Phi)^{2}\right)+\rho \gamma\left(e^{\Phi} \gamma-1\right)\right]_{t}
\end{array}
$$

Equation (2.19) is more useful than Eq. (2.17) because the leading-order conserved rest mass given by Eq. (2.10) has been subtracted off.

Another check on our numerical integrations is provided by the special relativistic virial theorem:

$$
\int T^{i j} d^{3} x=\frac{1}{2} \frac{d^{2}}{d t^{2}} \int T^{00} x^{i} x^{j} d^{3} x
$$

For systems that settle into an equilibrium state, the right-hand side of Eq. (2.20) vanishes. Taking the threedimensional trace of $T^{i j}$ on the left-hand side, and substituting Eq. (2.14), we find, in equilibrium,

$$
\begin{aligned}
\int \rho e^{\Phi} \gamma^{2} v^{2} d^{3} x & =\frac{1}{8 \pi G} \int(\nabla \Phi)^{2} d^{3} x \\
& =-\frac{1}{2} \int \rho \Phi e^{\Phi} d^{3} x
\end{aligned}
$$

To obtain the last equality we have integrated by parts and substituted Eq. (2.5), omitting the time derivative term. In the Newtonian limit, Eq. (2.21) reduces to the familiar result $2 K=-W$, where $K$ is the kinetic energy and $W$ is the gravitational potential energy of the system.

We can identify three "masses" in scalar gravitation theory. The first is rest mass defined in Eq. (2.10) and strictly conserved. The second is the total mass energy, given explicitly by the first integral in Eq. (2.17):

$$
\begin{aligned}
M & =\int T^{00} d^{3} x \\
& =\int\left[\frac{1}{8 \pi G}\left(\Phi_{, 0}^{2}+(\nabla \Phi)^{2}\right)+\rho e^{\Phi} \gamma^{2}\right] d^{3} x
\end{aligned}
$$

Provided we evaluate this at large enough radius, outside any radiation or matter, it is also conserved according to Eq. (2.17). For a static situation, the $\nabla \Phi$ term can be integrated by parts to give

$$
\begin{aligned}
M & =\int \rho e^{\Phi}\left(\gamma^{2}-\frac{1}{2} \Phi\right) d^{3} x \quad \text { (static) } \\
& \rightarrow \int \rho_{0}\left(1+\frac{1}{2} \Phi+\frac{1}{2} v^{2}\right) d^{3} x \quad \text { (Newtonian) }
\end{aligned}
$$

As we see, in the Newtonian limit $M$ is the sum of the rest mass plus the Newtonian energy.

Finally, we can define a "Coulomb mass":

$$
M_{C}=-\frac{1}{G} \lim _{r \rightarrow \infty} r \Phi \text {. }
$$

This mass determines the motion of distant particles, giving rise for example to Kepler's laws. In a static situation, we have, from Eq. (2.5), 


$$
\begin{aligned}
M_{C}= & -\int T d^{3} x \\
= & \int \rho e^{\Phi} d^{3} x \quad(\text { static }) \\
& \rightarrow \int \rho_{0}\left(1+\Phi-\frac{1}{2} v^{2}\right) d^{3} x \quad \text { (Newtonian) }
\end{aligned}
$$

Note that in general $\boldsymbol{M} \neq \boldsymbol{M}_{C}$, even in the Newtonian limit. This feature is in contrast with general relativity, where the total mass energy also determines the motion of distant particles.

\section{MONOPOLE RADIATION}

In the weak-field, slow-motion limit, the radiation field can be obtained by a multipole expansion. Since the theory involves a scalar field, the lowest-order contribution to the radiation comes from the monopole term. This is in contrast with electromagnetism (vector field: dipole radiation) or general relativity (tensor field: quadrupole radiation).

Using the usual Green's function for the wave equation, we can transform Eq. (2.5) into the integral form

$$
\Phi(t, \mathbf{x})=-G \int d^{3} x^{\prime} \frac{\left[e^{\Phi} \rho\right]_{\mathrm{ret}}}{\left|\mathbf{x}-\mathbf{x}^{\prime}\right|},
$$

where "ret" means evaluate at retarded time $t^{\prime}=t-\left|\mathbf{x}-\mathbf{x}^{\prime}\right|$. In the wave zone we can replace the denominator in Eq. (3.1) by the distance $r=|\mathbf{x}|$. To isolate the conserved rest mass $M_{0}$, define the rest density to be

$$
\rho_{0}=\gamma \rho \text {. }
$$

Then

$$
\Phi(t, \mathbf{x}) \approx-\frac{G}{r} \int d^{3} x^{\prime}\left[\frac{e^{\Phi}}{\gamma} \rho_{0}\right]_{\mathrm{ret}} .
$$

In the integrand, expand

$$
\begin{aligned}
\rho_{0}\left(t^{\prime}, \mathbf{x}^{\prime}\right)= & \rho_{0}\left(t-r, \mathbf{x}^{\prime}\right)+\left(r-\left|\mathbf{x}-\mathbf{x}^{\prime}\right|\right) \rho_{0, t} \\
& +\frac{1}{2}\left(r-\left|\mathbf{x}-\mathbf{x}^{\prime}\right|\right)^{2} \rho_{0, t t}+\cdots,
\end{aligned}
$$

and

$$
\frac{e^{\Phi}}{\gamma}=\left[1+\Phi-\frac{1}{2} v^{2}\right]_{t^{\prime}=t-r}+\cdots .
$$

For large $r$,

$$
r-\left|\mathbf{x}-\mathbf{x}^{\prime}\right| \approx \frac{\mathbf{x} \cdot \mathbf{x}^{\prime}}{r}=r^{\prime} \cos \theta^{\prime}
$$

The leading-order contribution to the expansion of Eq. (3.3) comes from the product of $\rho_{0}$ in Eq. (3.4) with the 1 in Eq. (3.5). The resulting integral gives $M_{0}$, so that this term represents the nonradiative Coulomb field. Thus the leading-order radiation field is

$$
\begin{aligned}
\Phi(t, \mathbf{x})=-\frac{G}{r} \int d^{3} x^{\prime}[ & \rho_{0}\left(\Phi-\frac{1}{2} v^{2}\right)+r^{\prime} \cos \theta^{\prime} \rho_{0, t} \\
+ & \left.\frac{1}{2} r^{\prime 2} \cos ^{2} \theta^{\prime} \rho_{0, t t}\right]_{t-r}
\end{aligned}
$$

To this order, it is irrelevant whether one use $\rho$ or $\rho_{0}$ in
Eq. (3.7).

For a spherically symmetric density distribution, the term proportional to $\cos \theta^{\prime}$ in Eq. (3.7) integrates to zero, giving

$$
\begin{aligned}
\Phi(t, r)=-\frac{G}{r} \int d r^{\prime} 4 \pi r^{\prime 2} & {\left[\rho_{0}\left(\Phi-\frac{1}{2} v^{2}\right)\right.} \\
& \left.+\frac{1}{6} r^{\prime 2} \rho_{0, t t}\right]_{t-r} \quad \text { (spherical). }
\end{aligned}
$$

This equation is the analogue of the "quadrupole formula" in general relativity. However, we see that, in contrast with general relativity, a scalar relativistic theory of gravitation can generate gravitational waves even in spherical symmetry. While a general angular-dependent solution of the vacuum scalar wave equation has terms that fall off like $1 / r, 1 / r^{2}, \ldots$, the spherically symmetric solution only has the $1 / r$ term. This means that in numerical simulations the wave amplitude generated by moving matter in spherical symmetry can be read off directly just outside the matter surface. Nonspherical waves in this scalar theory could also be read off just outside the matter: one simply "extracts" the $1 / r$ term from the higher-order terms. The behavior of these terms is exactly calculable from the field equation, which is linear in vacuum. In general relativity, where there are no spherically symmetric waves and where even the vacuum equations are nonlinear, wave extraction is more complicated [3].

From Eq. (3.8) we can easily estimate the typical size of $\Phi$ for a weak-field, slow-motion source of characteristic mass $M$, radius $R$ and velocity $v$ :

$$
\Phi \sim \frac{G M}{r c^{2}}\left(\frac{v}{c}\right)^{2} \sim \frac{G M}{r c^{2}} \frac{G M}{R c^{2}} .
$$

Here we have assumed the virial relation $v^{2} \sim G M / R$. The radiative energy flux in the wave zone is

$$
T_{0 r}^{\mathrm{field}}=-\frac{1}{4 \pi G} \Phi_{, t} \Phi_{, r}=\frac{1}{4 \pi G} \Phi_{, t}^{2} .
$$

Therefore the total rate of energy emission is

$$
\frac{d E}{d t}=4 \pi r^{2} T_{0 r}^{\mathrm{field}}=\frac{1}{G}\left(r \Phi_{, t}\right)^{2} .
$$

In order of magnitude, this gives

$$
\frac{d E}{d t} \sim \frac{c^{5}}{G}\left(\frac{v}{c}\right)^{10} \sim \frac{c^{5}}{G}\left(\frac{G M}{R c^{2}}\right)^{5} .
$$

Despite their very different origins, the wave amplitude and energy emission rate in Eqs. (3.10) and (3.12) are of the same magnitude as those given by the quadrupole formula in general relativity. However, for sources without equatorial symmetry, the second term in Eq. (3.7) dominates. This gives an amplitude larger than Eq. (3.10) by a factor $c / v$ and an emission rate larger than Eq. (3.12) by a factor of $c^{2} / v^{2}$. Because there is no angular dependence in the wave amplitude, this scalar radiation is still monopole in both cases. 


\section{SPHERICAL SYMMETRY}

Because scalar gravitation can generate gravitational waves in spherical symmetry, we can test out computational algorithms for calculating gravitational radiation on one-dimensional systems. This is much simpler than in general relativity theory. While not every aspect of the general relativistic problem is reflected in this setting, many features of wave generation in a rapidly varying nonlinear dynamical system are exhibited here.

In spherical symmetry, field equation (2.15) becomes

$$
\Phi_{, t t}=\frac{1}{r^{2}}\left(r^{2} \Phi_{, r}\right)_{, r}+4 \pi G T \text {. }
$$

The equation of motion (2.6) for each particle takes the form

$$
\begin{aligned}
& \frac{d r}{d t}=\frac{\widetilde{u}_{r}}{\widetilde{u}^{0}}, \\
& \frac{d \widetilde{u}_{r}}{d t}=\frac{\widetilde{u}_{\phi}^{2}}{\widetilde{u}^{0} r^{3}}-e^{2 \Phi} \frac{\Phi, r}{\widetilde{u}^{0}}, \\
& \widetilde{u}_{\phi}=\text { const },
\end{aligned}
$$

where

$$
\widetilde{u}^{0}=\left(e^{2 \Phi}+\widetilde{u}_{r}^{2}+\widetilde{u}_{\phi}^{2} / r^{2}\right)^{1 / 2},
$$

and where

$$
\widetilde{u}^{\alpha} \equiv e^{\Phi} u^{\alpha} \text {. }
$$

In this case, each particle moves in a plane, for which we choose $\theta=\pi / 2$, conserving its orbital angular momentum $\widetilde{u}_{\phi}$. For a static gravitational field, the particle energy $\widetilde{u}_{0}$ is also a constant.

\section{NUMERICAL METHOD}

Here we describe the scheme we use to solve the scalar field equation (4.1) coupled to the particle equations of motion (4.2)-(4.4). We focus on the spherical case because it is the simplest, yet it exhibits all the essential dynamical features including gravitational waves. The method that we adopt is a mean-field particle simulation scheme. A finite number of particles from the matter distribution are evolved in the mean background field $\Phi$ for a small time step $\Delta t$. From the new particle positions and velocities, we obtain the matter source term $T$ appearing in the field equation. We then update $\Phi$ by evolving the field equation for a time step $\Delta t$. The whole process is then repeated.

To solve Eq. (4.1) we employ a standard explicit leapfrog method, but we allow for a variable time step. We split the equation into two first-order equations by introducing the variable

$$
\lambda \equiv \Phi_{, t}
$$

Equation (4.1) now becomes

$$
\begin{aligned}
& \mathcal{T}[\Phi]=\lambda, \\
& \mathcal{T}[\lambda]=\mathscr{R}[\Phi]+4 \pi G T,
\end{aligned}
$$

where

$$
\begin{aligned}
& \mathcal{T}[Y] \equiv Y_{, t}, \\
& \mathcal{R}[Y]=6\left[r^{3} Y_{, r^{2}}\right]_{, r^{3}} .
\end{aligned}
$$

Here $Y$ denotes either $\Phi$ or $\lambda$. The Laplacian in the field equation has been written in the form shown in Eq. (5.3) to ensure regularity in the finite-difference operator near the origin (cf. Appendix of Ref. [4]). To finite difference the equations, introduce a radial grid $\left\{r_{i}\right\}$, $i=1,2, \ldots, i_{\max }$, extending from $r=0$ up to the outer boundary $r=r_{\max }$. The exact prescription for laying down the gridpoints will be discussed below. We take the fields $\Phi$ and $\lambda$ to be defined at the midpoint of radial zones. The finite difference form of the operators in Eq. (5.3) is then

$$
\begin{aligned}
& \mathcal{T}_{i+1 / 2}^{n}[Y]=\frac{\Delta t_{n-1}}{\Delta t_{n}+\Delta t_{n-1}} \frac{Y_{i+1 / 2}^{n+1}-Y_{i+1 / 2}^{n}}{\Delta t_{n}}+\frac{\Delta t_{n}}{\Delta t_{n}+\Delta t_{n-1}} \frac{Y_{i+1 / 2}^{n}-Y_{i+1 / 2}^{n-1}}{\Delta t_{n-1}}, \\
& \mathcal{R}_{i+1 / 2}^{n}[Y]=\frac{6}{r_{i+1}^{3}-r_{i}^{3}}\left[r_{i+1}^{3} \frac{Y_{i+3 / 2}^{n}-Y_{i+1 / 2}^{n}}{r_{i+3 / 2}^{2}-r_{i+1 / 2}^{2}}-r_{i}^{3} \frac{Y_{i+1 / 2}^{n}-Y_{i-1 / 2}^{n}}{r_{i+1 / 2}^{2}-r_{i-1 / 2}^{2}}\right),
\end{aligned}
$$

where $\Delta t_{n}=t_{n+1}-t_{n}$. These operators are second-order accurate in both space and time.

At $r=r_{\max }$ we impose an outgoing wave boundary condition

$$
(r Y)_{, t}+(r Y)_{, r}=0 \text {, }
$$

where $Y$ is either $\Phi$ or $\lambda$. A second-order accurate finite difference form of this equation is

$$
Y_{i_{\max }+1 / 2}^{n+1}=\frac{r_{i_{\max }-1 / 2}}{r_{i_{\max }+1 / 2}} Y_{i_{\max }-1 / 2}^{n}+\left(\frac{1-\zeta}{1+\zeta}\right)\left[Y_{i_{\max }+1 / 2}^{n}-\frac{r_{i_{\max }-1 / 2}}{r_{i_{\max }+1 / 2}} Y_{i_{\max }}^{n+1 / 2}\right) \text {, }
$$

where

$$
\xi=\frac{\Delta t_{n}}{r_{i_{\max }+1 / 2}-r_{i_{\max }-1 / 2}} .
$$

We do not need to explicitly impose a boundary condition at the origin: when evaluated for $i=1$, the term containing $Y_{1 / 2}$ in Eq. (5.4) is multiplied by $\left(r_{1}\right)^{2}=0$.

For a Cartesian grid uniform in space and time, the 
Courant stability condition for the above leapfrog scheme would be

$$
\Delta t<\frac{1}{2} \Delta r
$$

where $\Delta r=r_{i+1}-r_{i}$. Heuristically adapting this criterion to a variably spaced spherical polar grid with uneven time steps, we use the stability condition

$$
\Delta t=\frac{\alpha}{2} \min \left\{\Delta r_{i}\right\},
$$

where typically we choose $\alpha=0.5$ for accuracy. We find empirically that this criterion yields a stable scheme even when the grid is adjusted on each time step to follow the matter.

The matter term involving the trace $T$ appearing in Eq. (5.2) is computed from the particle distribution according to

$$
T=-\sum m_{A} n_{A} e^{\Phi}=-\sum m_{A}\left(n_{A} \gamma_{A}\right) \frac{1}{\widetilde{u}_{A}^{0}} e^{2 \Phi}
$$

where the comoving number density $n_{A}=\rho_{A} / m_{A}$ is given by

$$
n_{A}=\frac{1}{\mathcal{V}_{A}}=\frac{1}{4 \pi \gamma_{A} r^{2} \Delta r} \text {. }
$$

Here $\mathcal{V}_{A}$ is the comoving volume for particle $A$, and we treat the particle as smeared out over a zone size $\Delta r$. For evaluating the conservation equation (2.19), we also need two other matter source terms:

$$
\begin{aligned}
T_{\text {particle }}^{00} & =\sum m_{A} n_{A} \gamma_{A}^{2} e^{\Phi}=\sum m_{A}\left(n_{A} \gamma_{A}\right) \widetilde{u}_{A}^{0}, \\
T_{\text {particle }}^{0 r} & =\sum m_{A} n_{A} \gamma_{A}^{2} v_{r}^{A} e^{\Phi}=\sum m_{A}\left(n_{A} \gamma_{A}\right) \widetilde{u}_{r}^{A} .
\end{aligned}
$$

In order to obtain a smooth density profile from a discrete particle sample, we have experimented with several algorithms that share a given particle between several neighboring zones. In all cases we center the density on the midpoint of a zone, $r_{i+1 / 2}$. In our simplest scheme we assign a fraction of a particle's rest mass $m_{A}$ to the two zones whose centers surround the particle. The fraction given to each zone is proportional to the volume between the particle radius and each zone center. For particles near the origin, inside the first half zone, or near the surface, inside the last half zone, we assign their total weight to the nearest zone center.

In an alternative scheme we assign a fraction of a particle's rest mass to the nearest three zones according to the prescription of Hockney and Eastwood (Eqs. 5-88 of Ref. [5]). Their algorithm is derived for a onedimensional grid with uniform spacing and infinite extent. Since adjacent zones are almost equal in our scheme (see below), we can use their algorithm. We replace their linear distance coordinate with a spherical volume coordinate as in our simpler two-zone algorithm described above. Particles in the first and last zones would contribute to nonexistent adjacent zones. This contribution is assigned instead to the particle's own zone. An underlying principle in all these schemes is to conserve particle rest mass. We find that our simple scheme is adequate for tracking the gross motion of the particles, and for determining the overall shape of the wave form. However, the Hockney and Eastwood scheme gives a much smoother wave form, and is the one we generally use.

The particle equations of motion (4.2) are integrated in time by a fourth-order Runge-Kutta scheme with adaptive step size. The gradient of the potential is evaluated to second order by finite differencing $\Phi$ and interpolating to the particle position. While the time step for the fieldevolution equations is limited by the Courant condition (5.9), there is no stability limitation for the time step governing the particle equations. For accuracy, however, the particle coordinates must not change by a large fractional amount in a given step. For maximum efficiency, the code updates the field and particles separately, each with their own time step. Usually the field time step can be much shorter than the particle time step:

$$
\begin{aligned}
\frac{\Delta t^{\text {particle }}}{\Delta t^{\text {field }}} & \sim \frac{t_{\text {dyn }}}{\Delta t_{\text {Courant }}} \sim \frac{\left(R^{3} / G M\right)^{1 / 2}}{\Delta r_{\min } / c} \\
& \sim\left(\frac{R c^{2}}{G M}\right]^{1 / 2}\left[\frac{R}{\Delta r_{\min }}\right) \gg 1 .
\end{aligned}
$$

In practice, $\Delta t^{\text {particle }}$ must be a small fraction of the dynamical time $t_{\text {dyn }}$ for accuracy. To obtain very smooth wave forms, $\Delta t^{\text {particle }}$ must in fact be quite comparable to $\Delta t^{\text {field }}$.

A uniform grid spacing in $r$ is reasonably accurate. However, most configurations we are interested in are either roughly homogeneous or have a homogeneous central region. A mesh that is uniform in the volume coordinate $r^{3}$ in the region where $\rho \neq 0$ is then somewhat more accurate as it slices the configuration into roughly equal mass zones. In practice we set the first zone to follow a fixed fraction of the mass and then construct successive zones by geometric series to have volumes that vary by a fixed ratio, near unity. Outside the surface of the matter, we use a fixed number of zones to integrate out to $r=r_{\max }$. For smoothness at the surface, we make the first outer zone have the same width as the last inner zone. We then make the remaining outer zones form a geometric progression out to $r=r_{\max }$. For nonspherical fields it is necessary to set $r_{\max }$ much larger than the matter surface in order to implement the asymptotic boundary conditions accurately: Eq. (5.5) holds only for the leading-order $1 / r$ part of the field. For the special case of spherical symmetry, however, Eq. (5.5) holds even just outside the matter surface. Thus the outer radius can be very close to the matter surface.

In the weak-field, slow-motion limit, we can use the multipole expansion formula (3.8) as a check on our wave integration. We cast the equation into a sum over the particle positions and velocities. For a point particle, we can use the identity

$$
\int d^{3} x f(\mathbf{r}) \frac{d}{d t} \delta^{3}[\mathbf{r}-\mathbf{z}(t)]=\left.\frac{d f}{d t}\right|_{\mathbf{r}=\mathbf{z}(t)}
$$

to write 


$$
\int d r^{\prime} 4 \pi r^{\prime 2}\left[\frac{1}{6} r^{\prime 2} \rho_{0, t t}\right]=\int d r^{\prime} 4 \pi r^{\prime 2}\left[\frac{1}{6} \frac{d^{2}\left(r^{\prime 2}\right)}{d t^{2}} \rho_{0}\right]
$$

Using the equations of motion (4.2) in the weak-field limit, the right-hand side of Eq. (5.15) becomes

$$
\begin{aligned}
& \frac{1}{3} \int d r^{\prime} 4 \pi r^{\prime 2} \rho_{0}\left[\widetilde{u}_{r}^{2}+r \frac{d \widetilde{u}_{r}}{d t}\right] \\
& =\frac{1}{3} \int d r^{\prime} 4 \pi r^{\prime 2} \rho_{0}\left[\tilde{u}_{r}^{2}+\frac{\widetilde{u}_{\phi}^{2}}{r^{2}}-r \Phi_{, r}\right] .
\end{aligned}
$$

Thus Eq. (3.8) becomes

$$
\begin{aligned}
r \Phi(t, r)=G \sum m_{A} & {\left[\frac{1}{3} r_{a} \Phi_{, r_{A}}\right.} \\
& \left.-\Phi+\frac{1}{6}\left[\left(\widetilde{u}_{r}^{A}\right)^{2}+\frac{\left(\widetilde{u}_{\phi}^{A}\right)^{2}}{r_{A}^{2}}\right)\right]_{t-r} .
\end{aligned}
$$

Differentiating Eq. (5.17) with respect to time gives $\lambda$, and one can make substitutions using the equations of motion to simplify the resulting formula. We found instead that it was adequate to take numerical derivatives of Eq. (5.17) to get $\lambda$ for this check.

All the problems we have chosen to analyze start from a moment of time symmetry, so that

$$
\begin{aligned}
& \lambda=0, \\
& \nabla^{2} \Phi=4 \pi G e^{\Phi} \rho,
\end{aligned}
$$

subject to the boundary conditions

$$
\begin{aligned}
& \Phi_{, r}=0, \quad r=0, \\
& (r \Phi)_{, r}=0, \quad r \rightarrow \infty .
\end{aligned}
$$

Equation (5.18) is solved by iteration starting with an initial guess for $\Phi$ on the right-hand side. Finite differencing on our radial grid using the operator $\mathcal{R}$ in Eq. (5.4) then gives a simple tridiagonal system of linear equations that are iterated to convergence.

For the matter, we choose a random sample of particles to represent the initial density and velocity profiles. For sufficient resources, this scheme yields initial data of arbitrary accuracy even for strong fields and high particle velocities.

\section{ANALYTIC TEST}

In general, satisfying the conservation equation (2.19) provides a self-consistent check on a numerical integration. However, there is also a complete analytic solution that furnishes a good test for weak fields and slow velocities. The solution describes an oscillating homogeneous spherical cluster in Newtonian gravitation. Consider first a homogeneous equilibrium cluster of mass $M$ and radius $R_{0}$ consisting of particles moving in randomly oriented circular orbits about the cluster center. Now imagine that at $t=0$ all of the velocities are instantaneously reduced by a factor $\xi$. The resulting evolution consists of a periodic oscillation in which the cluster remains at all times homogeneous. The individual particles move in elliptic orbits with the same period and eccentricity, but with different semimajor axes. We first presented this model in Appendix C of Ref. [6], where we used it as a weak-field test of a general relativistic code. The radius $r$ of each particle satisfies

$$
r=r_{0} x(t)
$$

where $r_{0}$ is the initial radius and where $x(t)$ is given by the usual parametric equations for an elliptic orbit:

$$
\begin{aligned}
& x=a(1-e \cos u), \\
& t=\frac{P}{2 \pi}(u-e \sin u)-\frac{P}{2} .
\end{aligned}
$$

In Eq. (6.2), the semimajor axis, eccentricity and period are given by

$$
\begin{aligned}
& a=\frac{1}{2-\xi^{2}}, \\
& e=1-\xi^{2}, \\
& P=2 \pi\left[\frac{R_{0}^{3}}{G M\left(2-\xi^{2}\right)^{3}}\right]^{1 / 2} .
\end{aligned}
$$

The radial and tangential particle velocities are given by

$$
\begin{aligned}
& v_{r}=\frac{\dot{x}}{x} r \\
& v_{\phi}=\xi \frac{r}{x^{2}}\left(\frac{G M}{R_{0}^{3}}\right)^{1 / 2} .
\end{aligned}
$$

The cluster radius obeys

$$
R=R_{0} x \text {. }
$$

Inserting this analytic solution into Eq. (3.8) and differentiating with respect to time gives the wave amplitude

$$
r \lambda=-\frac{8}{5} \frac{(G M)^{2}}{R_{0}}\left[\frac{\dot{x}}{x^{2}}\right]_{t-r} \quad(\text { wave zone })
$$

From Eq. (3.11) we get, for the rate of energy emission,

$$
\frac{d E}{d t}=\frac{64}{25} \frac{(G M)^{4}}{R_{0}^{2}}\left[\frac{\dot{x}^{2}}{x^{4}}\right]_{t-r} .
$$

Integrating Eq. (6.7) over an oscillation period, we get, for the energy radiated per period,

$$
\begin{aligned}
\frac{\langle\Delta E\rangle_{\text {per period }}}{M c^{2}}= & \frac{64 \pi}{25}\left(\frac{G M}{R_{0} c^{2}}\right)^{7 / 2} \frac{\left(1-\xi^{2}\right)^{2}}{\xi^{7}} \\
& \times\left(\frac{5-2 \xi^{2}+\xi^{4}}{4}\right) .
\end{aligned}
$$




\section{NUMERICAL RESULTS}

When analyzing our numerical results, we will adopt gravitational units where $G=c=1$.

\section{A. Comparison with analytic model}

We have made extensive tests of our code employing the Newtonian analytic model of Sec. VI. Here we describe the case where the initial radius of the cluster is $R_{0} / M_{0}=500$ and the velocity cutdown factor is $\xi=0.7$. This choice of radius is sufficiently large that the system remains essentially Newtonian throughout its evolution, and sufficiently small that the required number of time steps is not too large [cf. Eq. (5.13)]. We set the outer grid at $r_{\max } / M_{0}=2500$. A typical run used $N_{P}=16000$ particles, 60 interior radial zones, and 180 exterior zones. Such a run requires 84000 time steps per oscillation period and takes about a second per time step on an IBM RS/6000 Model 550 workstation if the particles are advanced on each field time step [Eq. (5.9)]. The time essentially scales linearly with $N_{P}$.

As discussed in Sec. V, we do not really require an exterior grid extending far outside the matter surface. However, to test how well our code propagates waves in vacuum, we chose an unnecessarily large value for $r_{\max }$. Since most of the computer time is spent moving the particles, the additional zone coverage in the exterior has little overhead.

Figure 1 shows the evolution of the mean radius

$$
R_{\mathrm{rms}} \equiv\left(\frac{5}{3} \frac{1}{N_{P}} \sum r_{A}^{2}\right)^{1 / 2} \text {. }
$$

The factor of $5 / 3$ has been chosen so that the $R_{\text {rms }}$ will be exactly equal to the radius of a uniform density sphere represented by a homogeneous particle distribution. The agreement with the analytic result is excellent for this choice of resources.

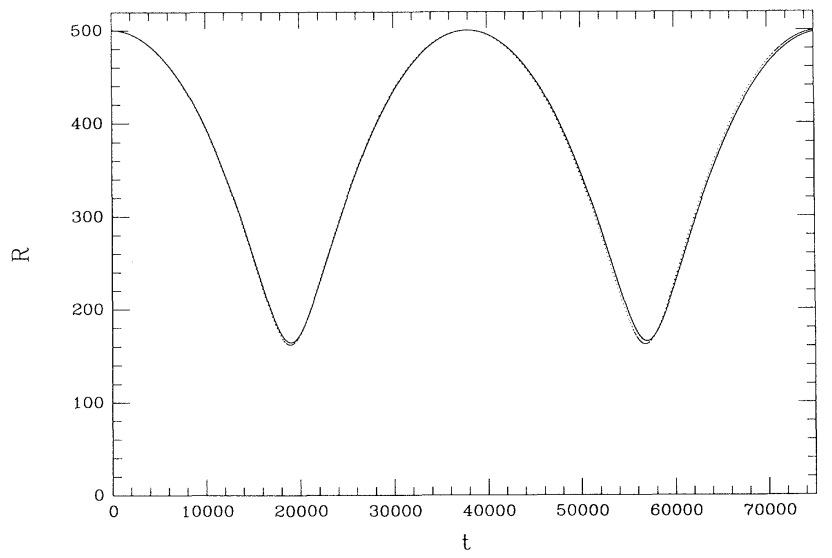

FIG. 1. Evolution of the mean radius as a function of time for a homogeneous Newtonian cluster with initial radius $R_{0} / M_{0}=500$ and $\xi=0.7$. The solid line shows the numerical result obtained from Eq. (7.1). The dotted line is the analytic result, Eq. (6.5). The two lines are virtually coincident in the plot. Both $R$ and $t$ are in units of $M_{0}$.
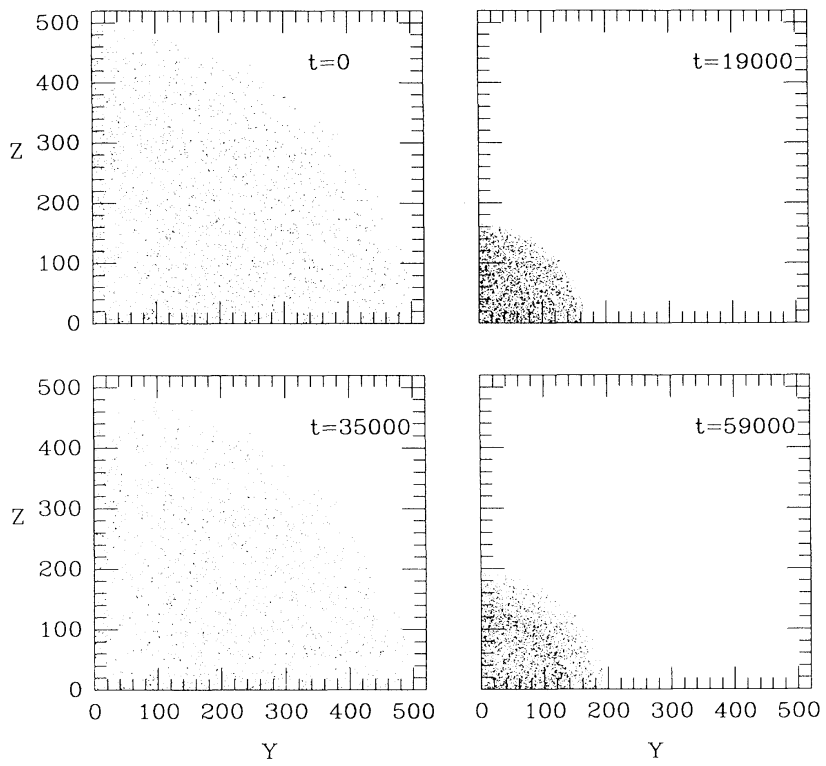

FIG. 2. Snapshots of the particle positions projected onto a plane through the origin for the evolution shown in Fig. 1. Particle coordinates and time are in units of $M_{0}$.

In Fig. 2 we show snapshots of the particle positions at selected times. Note how well the simulation maintains the homologous nature of the collapse, including the sharp boundary at the surface. We used Eq. (2.19) to monitor energy conservation at selected radii. At a radius $120 M_{0}$, which is always less than the minimum value of the radius $R(t)$, agreement is good to within a few percent when expressed as a fraction of the integrated flux. This agreement holds even at maximum compression where the conserved quantity involves the small

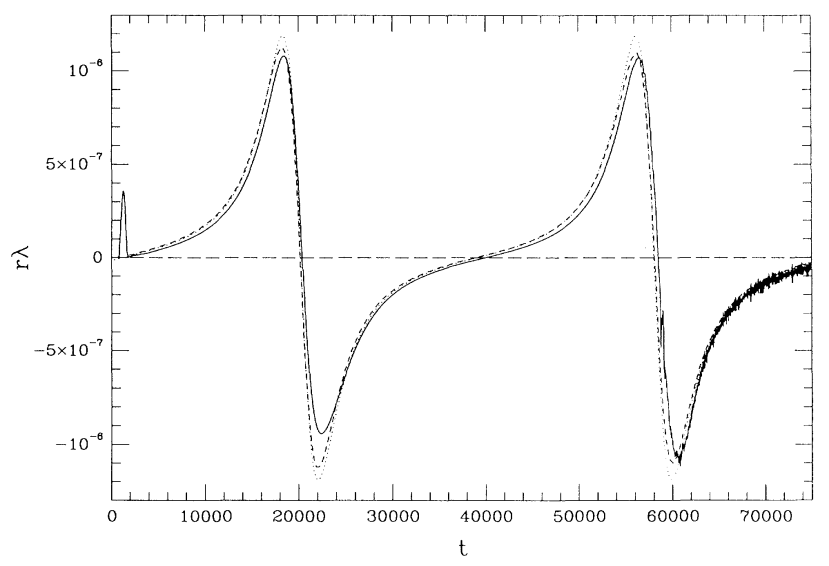

FIG. 3. Wave amplitude $\lambda$ (multiplied by $r$ ) as a function of time as measured at $r=250 M_{0}$ for the evolution shown in Fig. 1. The solid line shows the numerical result obtained from Eq. (5.2), the dashed line is the result from the multipole formula (5.17) numerically differentiated with respect to time, and the dotted line is the analytic result, Eq. (6.6). The small bump near $t=0$ is an artifact of slightly imprecise numerical initial data. $r \lambda$ is dimensionless, while $t$ is in units of $M_{0}$. 
difference of large numbers. The total Newtonian kinetic plus potential energy is conserved very well. By the end of the calculation it has changed by only $\frac{1}{2} \%$, while at maximum compression there is a momentary deviation of $1 \%$.

The emission of gravitational waves is a postNewtonian effect in the system. Figure 3 shows the wave amplitude $\lambda$ measured at a fixed exterior radius $r=250$. The amplitude agrees well with the monopole prediction obtained by numerically differentiating Eq. (5.17) with respect to time. Both of the amplitudes agree well with the analytic amplitude computed in Eq. (6.6) and also shown on the plot. We have verified that the agreement is even better when the system collapses from a larger radius and is therefore more Newtonian. Because the numerical simulation uses a finite number of particles, it cannot maintain the homogeneity of the system indefinitely. The first signs of the development of incoherent motion can be seen in the wave amplitude at late times in Fig. 3.

\section{B. Nonlinear homogeneous collapse}

To explore scalar gravitation in the nonlinear regime, we follow the same collapse described in Sec. VI A, except that we now choose the initial radius to be $R=3 M_{0}$. Specifically, the initial density is

$$
\rho_{0}=\frac{3 M_{0}}{4 \pi R^{3}},
$$

the initial radial velocity is zero, and the tangential velocity is given by

$$
\widetilde{u}_{\phi}^{2}=\xi^{2} r^{3} e^{2 \Phi} \Phi_{, r}
$$

If the cutdown factor $\xi$ is set equal to one, then the cluster is in equilibrium with each particle moving in a circular orbit [cf. Eq. (4.2)]. In general relativity, such an equilibrium system is called an Einstein cluster [7]. Here in scalar gravity we allow the system to collapse by setting $\xi=0.7$. For this choice of parameters, $M=0.88 M_{0}$ and $M_{C}=0.72 M_{0}$. We have previously considered analo-

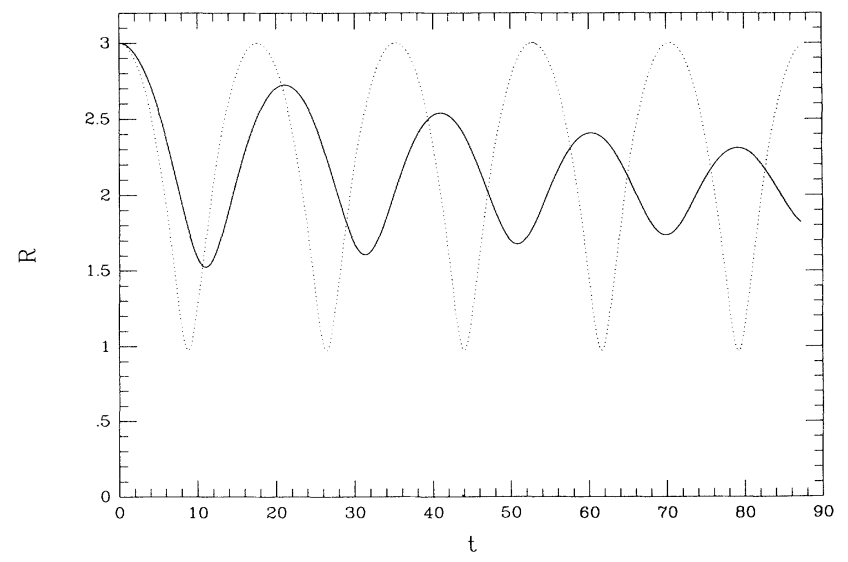

FIG. 4. Evolution of the mean radius as a function of time for a homogeneous cluster with initial radius $R_{0} / M_{0}=3$ and $\xi=0.7$. Labeling is the same as in Fig. 1 . gous strong field collapses in general relativity [6].

In Fig. 4 we plot the mean radius of the system as a function of time. From the figure we see that the oscillations are slowly damped because the nonlinear field causes departures from strict homogeneity and homologous behavior. This effect signals the onset of phase mixing and "violent relaxation," which ultimately drives the system to virial equilibrium. Note the large difference between the nonlinear evolution and the behavior predicted from the analytic Newtonian model.

In Fig. 5 we show snapshots of the particle positions at selected times during the evolution. The departures from homogeneity grow slowly in this example, but are discernible in the figure.

Since there is no analytic solution for this case, the only check we have is provided by the energyconservation equation (2.19). We show how well the code conserves energy in Fig. 6. We evaluate the conservation equation as a function of time at three fixed radii. The first radius is inside the matter at all times; the second is alternately inside and outside the matter as the surface oscillates back and forth; the third radius is always in the vacuum exterior. In the first two cases the integrated flux term (second integral in Eq. 2.19) is large, and energy conservation involves the small difference of large terms. Consequently, the high degree to which we are able to maintain energy conservation is a nontrivial measure of the accuracy of the code. In the exterior, the flux is small and energy conservation is not a stringent test. However, the wave propagates according to the linear scalar wave equation in the exterior, and the analytic test at small wave amplitude already validated this aspect of the code.

The wave amplitude at fixed radius for this case is plotted as a function of time in Fig. 7. By contrast with the Newtonian example, the wave amplitude in this strong-
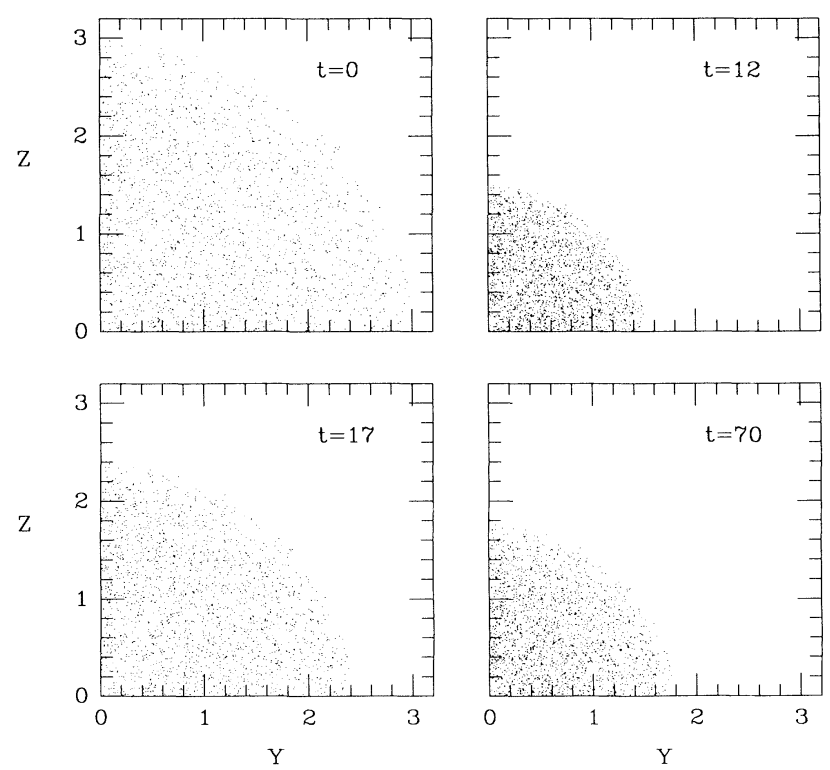

FIG. 5. Snapshots of the particle positions projected onto a plane through the origin for the evolution shown in Fig. 4. Particle coordinates and time are in units of $M_{0}$. 
field case is not adequately described by the weak-field multipole expansion formula (5.17). Moreover, the amplitude is slowly damped as the matter oscillations decay. Figure 8 shows a snapshot of the amplitude as a function of radius at fixed time. The damping is clearly evident in the exterior wave form. Note the sharp transition be-
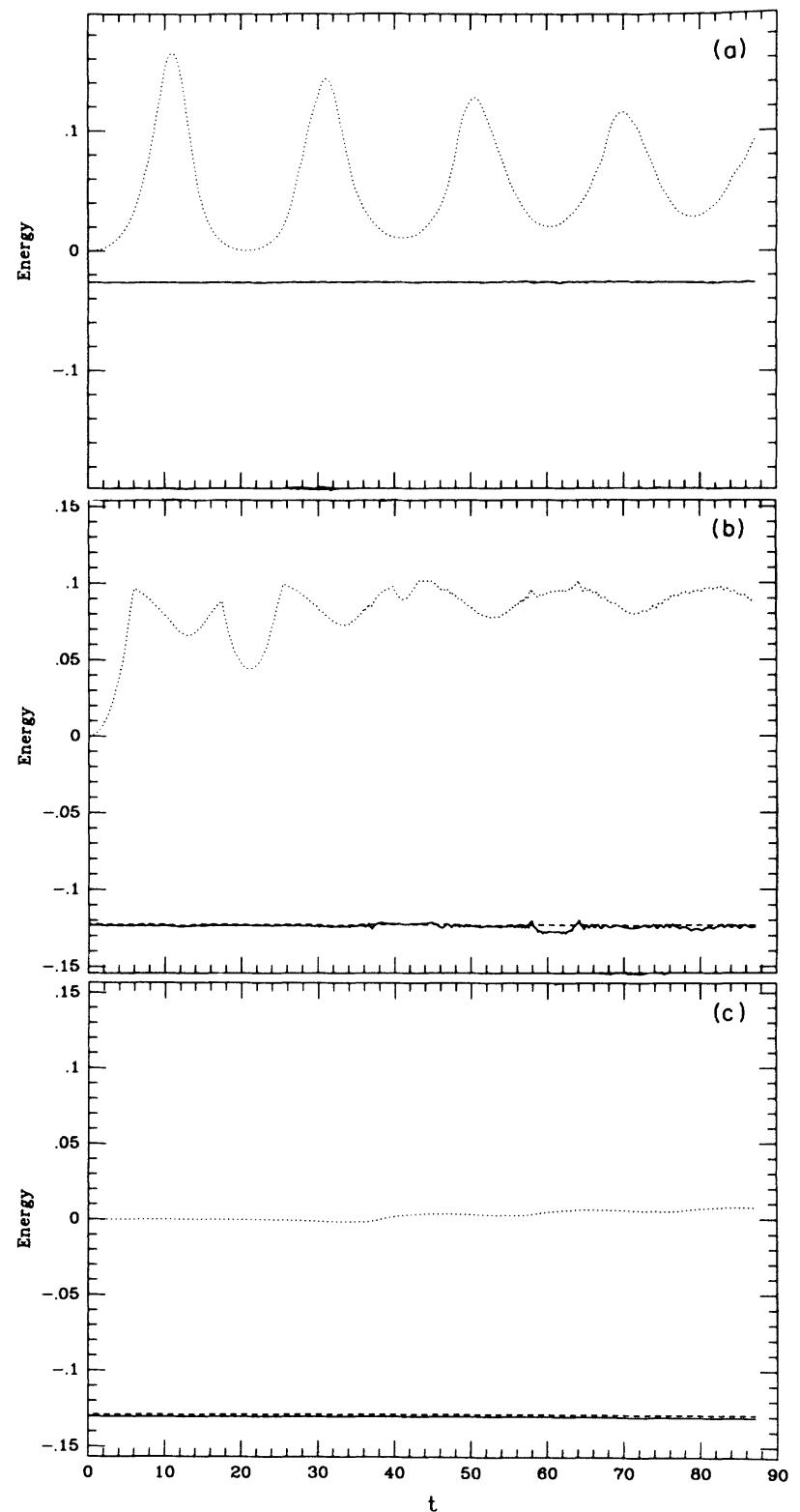

FIG. 6. Energy conservation at three selected radii as a function of time for the evolution described in Fig. 4. The solid line shows the left-hand side of Eq. (2.19) (volume integral plus integrated flux), the dotted line shows the second term alone (integrated flux), and the dashed line shows the right-hand side (volume integral at $t=0$ ). The radii are (a) $r=1.4 M_{0}$, (b) $r=2.4 M_{0}$, (c) $r=25 M_{0}$. Energy and time are in units of $M_{0}$. The degree to which the solid and dashed lines coincide compared with the magnitude of the dotted line is a measure of the code's ability to conserve energy.

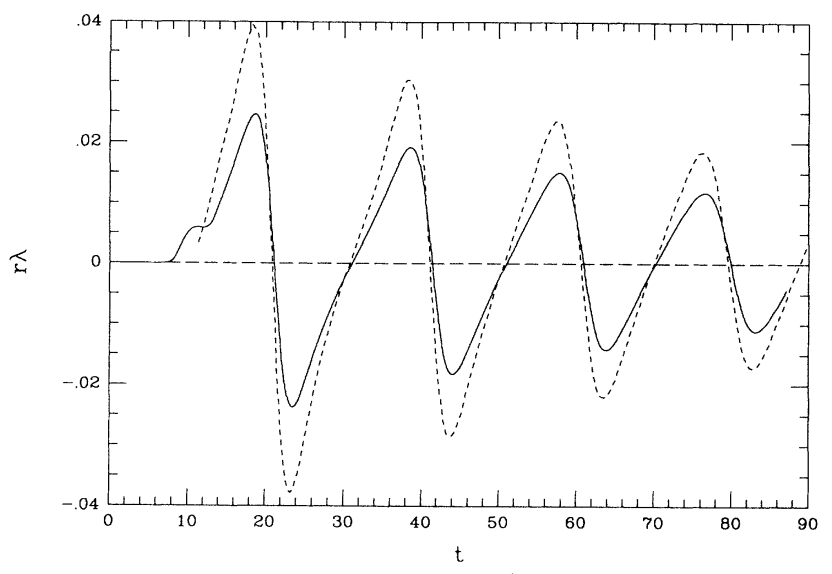

FIG. 7. Wave amplitude $\lambda$ (multiplied by $r$ ) as a function of time as measured at $r=10.5 M_{0}$ for the evolution shown in Fig. 4. Labeling is the same as in Fig. 3.

tween the near zone and far zone fields at the matter surface.

\section{Inhomogeneous collapse}

We have seen that initially homogeneous systems oscillate rather homologously; even when they are nonlinear, the oscillations persist for many cycles. We do not expect this behavior for inhomogeneous configurations. In this case the incoherent motions tend to damp the oscillations rapidly through violent relaxation. The system is driven towards virial equilibrium after only a few cycles. If an inhomogeneous system is centrally condensed, then another distinction may be relevant computationally: the absence of a density discontinuity at the surface.

To explore these differences, we followed the collapse of a centrally condensed system with an initial density profile given by

$$
\rho_{0}=\frac{M_{0}}{4 \pi R^{3}} \frac{105}{8}\left[1-\frac{r^{2}}{R^{2}}\right]^{2},
$$

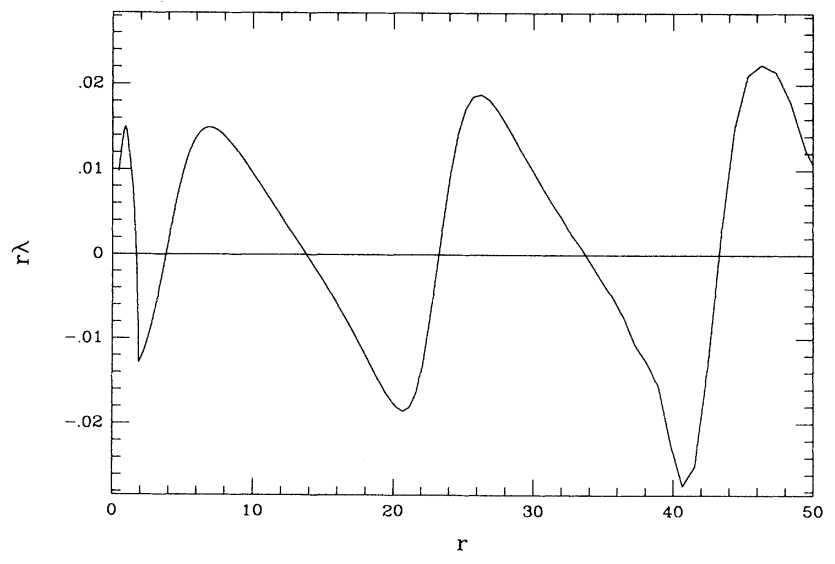

FIG. 8. Snapshot of the wave amplitude $\lambda$ (multiplied by $r$ ) as a function of $r$, as measured at $t=54 M_{0}$. 


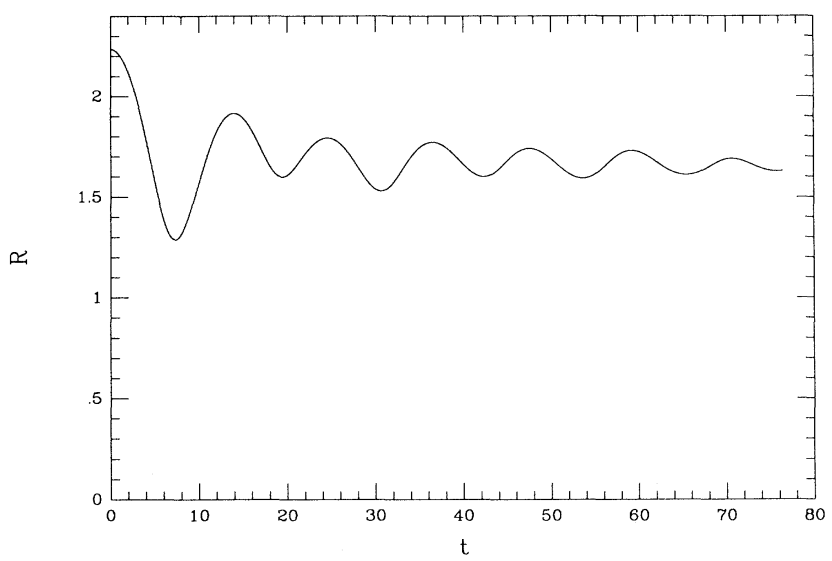

FIG. 9. Evolution of the mean radius as a function of time for an inhomogeneous cluster (Eq. (7.4) with initial radius $R / M_{0}=3$ and $\xi=0.7$. Labeling is the same as in Fig. 1.

and an initial tangential velocity profile given by Eq. (7.3), with $\xi=0.7$. We consider collapse from a radius $R=3 M_{0}$ as in the homogeneous case discussed above. For this case, $M=0.83 M_{0}$ and $M_{C}=0.67 M_{0}$.

Figure 9 shows the evolution of the mean radius, which clearly shows the damped oscillations. Because of the initial inhomogeneity, the damping is much more pronounced than in the corresponding homogeneous case, Fig. 4. Snapshots of the particle positions are shown in Fig. 10. Following an initial implosion, the bulk of the mass settles down in a new equilibrium state. The resulting configuration displays the typical core-halo structure characteristic of inhomogeneous gravitational collapse and virialization. The wave amplitude, plotted in Fig. 11, shows an initial burst followed by damped oscillations of smaller magnitude. As shown in the plot, at late times
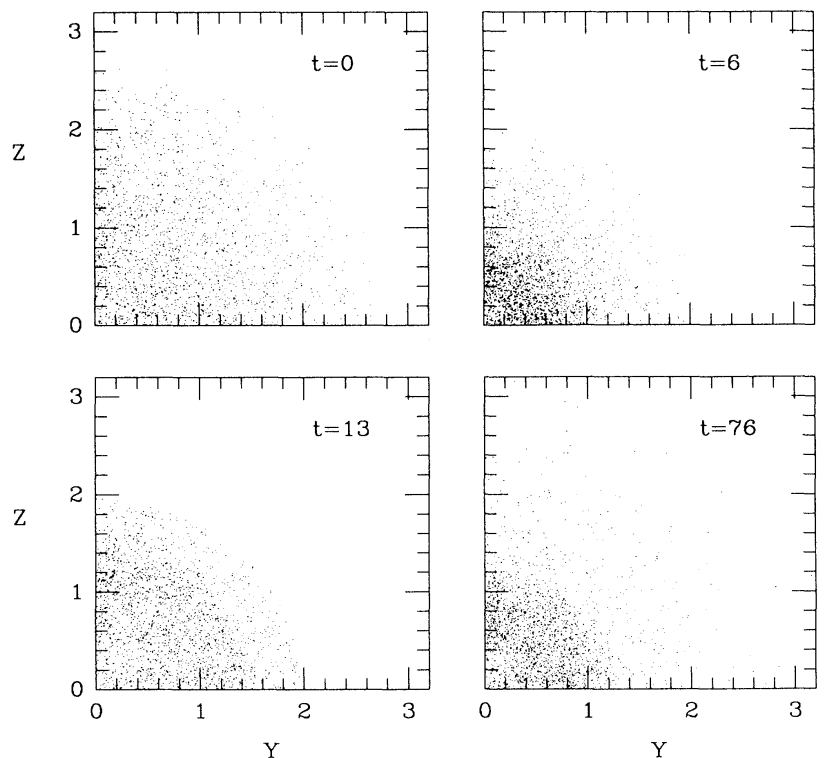

FIG. 10. Snapshots of the particle positions projected onto a plane through the origin for the evolution shown in Fig. 9.

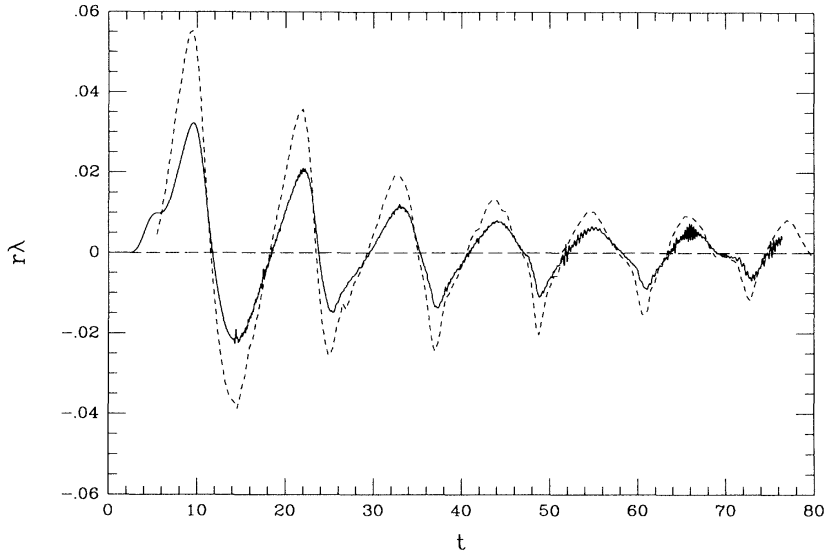

FIG. 11. Wave amplitude $\lambda$ (multiplied by $r$ ) as a function of time as measured at $r=5 M_{0}$ for the evolution shown in Fig. 9. Labeling is the same as in Fig. 3.

the wave amplitude exhibits small scale fluctuations about its mean value. We are able to damp these highfrequency components reliably by adding a small KreissOliger [8] diffusive term to the leapfrog scheme (5.4).

\section{CONCLUSIONS}

Scalar gravitation is by no means a correct physical theory, but it provides a perfectly fine and simple framework for learning how to do numerical relativity. Because the theory allows gravitational waves to be generated in spherical symmetry, one can try out methods of calculating wave emission and propagation in only one spatial dimension. We have used the theory to test out a mean-field particle simulation scheme for the dynamical evolution of collisionless matter in a self-consistent gravitational field. We find that the scheme allows one to calculate smooth and accurate gravitational wave forms. The stochastic representation of the particle distribution is not an inherent obstacle. With sufficient computer resources, a similar scheme should be able to calculate reliable gravitational wave amplitudes in general relativity.

\section{ACKNOWLEDGMENTS}

This research was supported in part by NSF Grants Nos. AST 90-15451 and PHY 90-07834 and NASA Grant No. NAGW-2364 at Cornell University.

\section{APPENDIX}

For a perfect fluid, the appropriate stress-energy tensor for the matter is

$$
T_{\mu \nu}^{\text {fluid }}=e^{\Phi}\left[(\epsilon+P) u_{\mu} u_{v}+P g_{\mu \nu}\right],
$$

where $\epsilon$ is the total mass-energy density of the fluid and $P$ is the pressure. Equation (2.15) now takes the form

$$
\square \Phi=4 \pi G e^{\Phi}(\epsilon-3 P) \text {. }
$$

To get the fluid equation of motion, introduce the projection tensor 


$$
P_{\alpha \mu}=u_{\alpha} u_{\mu}+g_{\alpha \mu} .
$$

Then in Cartesian coordinates

$$
P_{\alpha \mu} T_{, v}^{\mu v}=0 \text {, }
$$

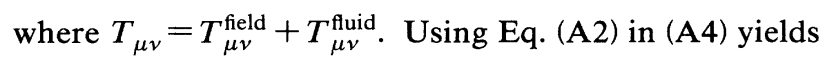

$$
(\epsilon+P) u_{\alpha, v} u^{v}=-P_{\alpha}^{v}\left[P_{, v}+\Phi_{, v}(\epsilon-2 P)\right],
$$

which is the relativistic Euler equation in this theory.

The fluid energy equation follows from

$$
u_{\mu} T_{, v}^{\mu v}=0 \text {, }
$$

which gives

$$
\frac{d \epsilon}{d \tau}=-(\epsilon+P) \nabla \cdot \vec{u}-3 P \frac{d \Phi}{d \tau} .
$$

Using the continuity equation

$$
\nabla \cdot(n \vec{u})=0,
$$

where $n$ is the comoving baryon density, we can rewrite Eq. (A7) as

$$
d \epsilon=\frac{\epsilon+P}{n} d n-3 P d \Phi .
$$

In this theory, Eq. (A9) is the first law of thermodynamics for a perfect fluid in an external scalar potential $\Phi$.

In stationary flow, the fluid equations yield a Bernoulli integral. Dotting the four-vector $\vec{\xi}=\partial / \partial t$ into Eq. (A5) and substituting Eq. (A9), we find

$$
u_{0} \frac{\epsilon+P}{n} e^{\Phi}=\text { constant along streamline }
$$

[1] C. W. Misner, K. S. Thorne, and J. A. Wheeler, Gravitation (Freeman, San Francisco, 1973).

[2] S. L. Shapiro and S. A. Teukolsky, Phys. Rev. Lett. 66, 994 (1991); Am. Sci. 79, 330 (1991).

[3] A. M. Abrahams and C. R. Evans, Phys. Rev. D 42, 2585 (1990).

[4] S. L. Shapiro and S. A. Teukolsky, Astrophys. J. 318, 542 (1987).

[5] R. W. Hockney and J. W. Eastwood, Computer Simulation
Using Particles (McGraw-Hill, New York, 1981).

[6] S. L. Shapiro and S. A. Teukolsky, Astrophys. J. 298, 34 (1985).

[7] A. Einstein, Ann. Math. (N.Y.) 40, 922 (1939).

[8] H. O. Kreiss and J. Oliger, Methods for the Approximate Solution of Time Dependent Problems, GARP Publication Series No. 10 (World Meterological Organization, Geneva, 1973). 